\title{
Delayed Inflammatory Reactions to Hyaluronic Acid Fillers: A Literature Review and Proposed Treatment Algorithm
}

This article was published in the following Dove Press journal:

Clinical, Cosmetic and Investigational Dermatology

Ofir Artzi, (iD ${ }^{1,2}$ Joel L Cohen, ${ }^{3}$ Jeffrey $S$ Dover, ${ }^{4-6}$ Atchima Suwanchinda, ${ }^{7,8}$ Tatjana Pavicic, ${ }^{9}$ Marina Landau, (iD ${ }^{10}$ Greg J Goodman, (D) " Sahar Ghannam, ${ }^{12}$ Firas Al Niaimi, ${ }^{13}$ Jani AJ van Loghem, ${ }^{14}$ Kate Goldie, ${ }^{15}$ Sonja Sattler, ${ }^{16}$ Daniel Cassuto, (D) ${ }^{17}$ Ting Song Lim, ${ }^{18}$ Rungsima Wanitphakdeedecha, ${ }^{19}$ Ines Verner, (iD) ${ }^{20,21}$ Tanja C Fischer, (iD ${ }^{22,23}$ Vivian Bucay, (iD) ${ }^{24}$ Eli Sprecher, ${ }^{1,2,25}$ Dana Shalmon ${ }^{1,2}$

'Department of Dermatology, Tel Aviv Sourasky Medical Center, Tel Aviv, Israel; '2Sackler Faculty of Medicine, Tel Aviv University, Tel Aviv, Israel;

${ }^{3}$ AboutSkin Dermatology and AboutSkin Research, , Greenwood Village and Lone Tree, CO, USA;

${ }^{4}$ SkinCare Physicians, Chestnut Hill, MA, USA;

${ }^{5}$ Dermatology, Yale University School of Medicine, New Haven, CT, USA; ${ }^{6}$ Dermatology, Brown Medical School, Rhode Island, USA; ${ }^{7}$ Department of Dermatology, School of Anti-Aging and Regenerative Medicine, Mae Fah Luang University, Bangkok, Thailand; ${ }^{8}$ Division of Dermatology, Faculty of Medicine, Ramathibodi Hospital, Mahido University, Bangkok, Thailand; ${ }^{9}$ Private Practice for Dermatology and Aesthetics Dr. Tatjana Pavicic, Munich 80539, Germany; ${ }^{10}$ Dermatology, Private Practice, Herzliya, Israel; "'Monash University, Clayton, Victoria, Australia; ${ }^{12}$ Associate Prof. of Dermatology, Alexandria University, Alexandria,

Egypt; ${ }^{13}$ I52, Harley street, London, UK; ${ }^{14}$ UMA Institute, Amsterdam 1017, TX, Netherlands;

${ }^{15}$ Medical Director European Medical Aesthetics Ltd, London WIG 8QN, UK; ${ }^{16}$ Rosenpark Klinik, Darmstadt, Germany; ${ }^{17}$ Private Practice, Milan, Italy; ${ }^{18}$ Clique Clinic, Kuala Lumpur, Malaysia; ${ }^{19}$ Department of Dermatology, Faculty of Medicine, Siriraj Hospital, Mahidol University, Bangkok, Thailand; ${ }^{20}$ Verner Clinic, Tel Aviv, Israel; ${ }^{21}$ Department of Dermatology, University of Rome, Guglielmo Marconi, Italy; ${ }^{22}$ Skin and Laser Center, Potsdam, Germany; ${ }^{23}$ School of Medicine, University of Frankfurt, Germany; ${ }^{24}$ Bucay Center for Dermatology and Aesthetics, UTHSC, San Antonio, TX, USA; ${ }^{25}$ Department of Human Molecular Genetics \& Biochemistry, Sackler Faculty of Medicine, Tel Aviv University, Tel Aviv, Israel

Correspondence: Ofir Artzi

Tel Aviv Sourasky Medical Center, Weizman 6 , Tel Aviv, Israel

Tel +972-54-596896।

Fax +972-77-200180I

Email ofira@tlvmc.gov.il
Background and Objectives: There is a wide diversity of opinions regarding the management of delayed inflammatory reactions (DIRs) secondary to hyaluronic acid (HA)-based fillers. The plethora of approaches has led the authors to conduct a review regarding management and treatment of DIRs as well as establish therapeutic guidelines for this purpose.

Materials and Methods: A review of the literature was performed through databases such as PubMed using keywords including HA-fillers and complications, delayed HA filler sequelae and therapy, soft tissue and dermal filler reactions and management. Additionally, a survey comprised of questions regarding the management and treatment of DIRs was sent to 18 physicians highly experienced with soft-tissue filler injections in 10 countries. Their answers and recommendations were analyzed and debated amongst these panelists.

Results: Sixteen panelists favored antibiotic therapy as first-line treatment for DIRs, specifically dual antibiotic therapy consisting of a fluoroquinolone along with a tetracycline or macrolide for a period of 3-6 weeks. The majority refrained from the use of intralesional (IL) or systemic steroids except in the case of disfiguring or recalcitrant reactions. IL hyaluronidase was recommended by 13 panelists; however, some preferred a watchful waiting approach for a period of 48 hours to 2 weeks prior to IL hyaluronidase, and in cases where antibiotics did not lead to improvement.

Conclusion: A consensus was reached and summarized to propose a clear, easy-to-follow, stepwise algorithm for the treatment of DIRs.

Keywords: cosmetic techniques, dermal fillers/adverse effects, hyaluronic acid/adverse effects

\section{Introduction}

Delayed inflammatory reactions (DIRs) to hyaluronic acid (HA)-based fillers are widely discussed and debated at many conferences as well as in the scientific literature. A recent Israeli survey illustrated extensive variations in DIRs definition and management protocols. ${ }^{1}$ The aim of the current study is to provide a clear, easy-to-follow, stepwise approach to the therapeutic management of DIRs.

\section{Methods}

A questionnaire comprising open questions on the management of DIRs was sent to 18 physicians highly experienced with soft-tissue filler injections in 10 countries. The research questionnaire was approved by the Tel Aviv Medical Center institutional review board in accordance with the principles of the Declaration of Helsinki and the 
participants' consent was provided in a written form. The first questions focused on demographic data, including the responders' specialties, number of years they had been performing HA-based filler injections, number of $1 \mathrm{~mL}$ syringes used per week, and the average number of DIRs they encountered in a year. The second part of the questionnaire aimed at assessing their approach to DIRs management. The physicians were asked to specify treatment options, including oral antibiotics, oral corticosteroids, intralesional (IL) corticosteroids, non-steroidal anti-inflammatory drugs (NSAIDs), IL hyaluronidase, IL 5-fluorouracil (5-FU), topical therapy with a corticosteroid or calcineurin inhibitor, laser therapy, and excision. They were also asked to provide the exact name of the drug/drug combination, dose, and duration of treatment, and to add any pearls and tips they would recommend. Finally, they were asked to propose a stepwise, easy-to-follow algorithm containing specific first-line and second-line recommendations. The last three questions dealt with recurrent DIR episodes, subsequent filler injections following such reactions, as well as precautions taken to avoid DIRs.

\section{Results}

Eighteen physicians including 14 dermatologists, 1 plastic surgeon and 3 aesthetic physicians responded to the questionnaire. Missing data or information were filled-in by direct correspondence to the specific physicians. The average number of years of experience with HA-based filler injections was $17.88 \pm 5.86$ (range: 7-29). The average number of injected HA-filled $1 \mathrm{~mL}$ syringes per week was $46.83 \pm 25.43$ (range: 15-90). The average number of DIRs per year was $6.94 \pm 4.59$ (range: $2-15$ ).

Table 1 provides a summary of the panelists' questionnaire answers. Sixteen out of the 18 expert panelists agreed that antibiotics should be given as first-line therapy, and 11 of them believed that dual antibiotic therapy should be used, whereas five favored monotherapies. Among those who preferred monotherapy, the antibiotic of choice was a tetracycline, a macrolide, or a fluoroquinolone. Those antibiotics were chosen due to their broadspectrum activity as well as their anti-inflammatory properties. Moreover, panelists who supported the use of one antibiotic regarded dual antibiotic therapy as more likely to cause pseudomembranous colitis. First-line antibiotic monotherapy treatment regimens included doxycycline $100 \mathrm{mg}$ daily for $1 \mathrm{month}$, minocycline $100 \mathrm{mg}$ daily for 2-4 weeks, azithromycin $500 \mathrm{mg}$ TID for 3-6 weeks, or amoxicillin/clavulanic acid $875 \mathrm{mg}$ BID for 2-4 weeks. Of note, three responders emphasized the concomitant use of probiotics when they prescribed long-term antibiotic treatment. Furthermore, three physicians rejected the use of clindamycin or amoxicillin/clavulanic acid based on the risk of developing bacterial resistance. Panelists who supported the use of dual antibiotic therapy recommended combining a fluoroquinolone (ie, ciprofloxacin) with either a tetracycline (ie, minocycline) or a macrolide (ie, clarithromycin, azithromycin). Fluoroquinolones, macrolides, and tetracyclines were recommended due to their antiinflammatory properties. First-line dual antibiotic treatment approaches included a regimen of ciprofloxacin $500 \mathrm{mg}$ BID + minocycline $100 \mathrm{mg}$ daily or clarithromycin $500 \mathrm{mg}$ BID for 3-6 weeks, or azithromycin $500 \mathrm{mg}$ daily for 3 days per week (and 4 days off) in conjunction with levofloxacin $500 \mathrm{mg}$ daily for 5 days per week (and 2 days off) for a period of 3-6 weeks. One of the panelists recommended first-line dual antibiotic therapy consisting of a 14-day course of clindamycin (300 mg TID) with ciprofloxacin (500 $\mathrm{mg}$ BID). One of the panelists did not support the use of fluoroquinolones based on the risk of tendon rupture as well as on the evidence in recent reports of rupture of aortic aneurysms. ${ }^{26 .}$

Systemic corticosteroid treatment of DIRs stirred considerable debate among the panelists. Five of the 18 participating physicians advised avoiding the use of corticosteroids altogether in the treatment of DIRs. Masking of symptoms, rebound of infection upon discontinuation of corticosteroids, dependence upon systemic corticosteroid use, as well as the well-documented side effects of systemic corticosteroids were the reasons most cited. Only three panelists supported treatment with a lowdose $(0.5-0.75 \mathrm{mg} / \mathrm{kg})$ or a short-medium course of systemic corticosteroid as first-line therapy (eg, a course of prednisone $40 \mathrm{mg}$ daily for 7-21 days followed by tapering). Ten panelists considered that the role of systemic corticosteroids in the management of DIRs depended on the severity and the extent of the inflammatory reaction and reserved the use of corticosteroids to overwhelming and disfiguring inflammatory reactions or for recalcitrant cases. One panelist favored only short-term use of 20-40 mg prednisolone for 3 days with no tapering but in combination with antibiotics. Eleven of the physicians agreed that the use of gastric protectors (H2-blockers or proton pump inhibitors) was indicated when administering systemic corticosteroid therapy.

IL 5FU alone or in combination with IL corticosteroids as first-line therapy was supported by two of the 
Table I Panelist Survey Response Summary

\begin{tabular}{|c|c|c|c|c|}
\hline & Antibiotics & Corticosteroids & Hyaluronidase & $\begin{array}{l}\text { 5-Fluorouracil } \\
\text { (5-FU) }\end{array}$ \\
\hline \multirow[t]{3}{*}{ First Line } & In Favor: 16/18 & Avoid: 5/18 & In favor: $13 / 18$ & Avoid: $1 / 18$ \\
\hline & $\begin{array}{l}\text { I I//8 dual } \\
\text { therapy: } \\
\text { fluoroquinolone } \\
+ \text { tetracycline } \\
\text { OR } \\
\text { fluoroquinolone } \\
+ \text { macrolide } \\
\text { for } 3-6 \text { weeks }\end{array}$ & \multirow{2}{*}{$\begin{array}{l}\text { 3//8: } \\
\text { Low dose }(0.5-0.75 \mathrm{mg} / \mathrm{kg}) \\
\text { OR } \\
\text { short-medium course eg Prednisone } \\
40 \mathrm{mg} / \text { day for } 7-2 \text { I days (followed by } \\
\text { tapering) } \\
\text { OR } \\
\text { short-term Prednisolone ( } 20-40 \mathrm{mg} / \\
\text { day for } 3 \text { days) without tapering and } \\
\text { combined with antibiotics }\end{array}$} & \multirow[t]{2}{*}{$\begin{array}{l}\text { 3/I3: } \\
\text { Watchful waiting for } 48 \text { hours to } 2 \text { weeks } \\
\text { prior to IL hyaluronidase, and when } \\
\text { antibiotics did not lead to improvement }\end{array}$} & \multirow[t]{2}{*}{$\begin{array}{l}\text { 2/I8: } \\
\text { Intralesional (IL) } 5-\mathrm{FU} \\
\text { alone or in } \\
\text { combination with IL } \\
\text { steroids }\end{array}$} \\
\hline & $\begin{array}{l}\text { 5/18 } \\
\text { Monotherapy: } \\
\text { tetracycline/ } \\
\text { macrolide/ } \\
\text { fluoroquinolone } \\
\text { for 2-6 weeks }\end{array}$ & & & \\
\hline \multirow{2}{*}{$\begin{array}{l}\text { Second Line } \\
\text { OR } \\
\text { Recalcitrant } \\
\text { Cases }\end{array}$} & & Avoid: 5/18 & \multirow{2}{*}{$\begin{array}{l}\text { 2//8: } \\
\text { Advise against repeating IL hyaluronidase } \\
\text { after two treatments every } 2 \text { weeks }\end{array}$} & \multirow{2}{*}{$\begin{array}{l}\text { I5/I8: } \\
\text { IL 5-FU every } 2-4 \\
\text { weeks till resolution }\end{array}$} \\
\hline & & $\begin{array}{l}\text { 10/I8: reserve for overwhelming and } \\
\text { disfiguring or recalcitrant cases }\end{array}$ & & \\
\hline
\end{tabular}

panelists, one panelist rejected their use, and the remaining 15 recommended their use every $2-4$ weeks until resolution for recalcitrant cases. The use of IL hyaluronidase for soft-tissue filler removal as first-line therapy was favored by 13 of the 18 panelists. However, three of those 13 advised watchful waiting from 48 hours to 14 days prior to the injection of hyaluronidase and to use it only when the initial course of antibiotics did not lead to improvement. Two panelists stated they would not repeat IL hyaluronidase injections more than twice every 2 weeks. Those in favor of using hyaluronidase reasoned that its use would help break down the cluster of hyaluronic acid and the surrounding inflammatory matrix in order to potentially increase the efficacy of the antibiotic therapy. Five panelists refrained from the use of IL hyaluronidase as first-line therapy in order to maintain the benefit of the soft-tissue filler. The hyaluronidase dosage was also an issue of debate among the panelists. Ten panelists recommended the use of 50-250 units or more if needed, depending upon the size and number of the DIRs, whereas five advised using 30 units of hyaluronidase only 24-48 hours after commencing antibiotic therapy.

Sixteen panelists agreed that they would treat recurrent episodes in the same manner as they would an initial episode. Four panelists suggested switching from antibiotics to systemic corticosteroids in the event of a relapsing episode.

\section{Discussion}

The incidence of adverse events has escalated in parallel to the continuing rise in popularity of soft-tissue filler procedures. Different HA-based fillers are associated with different incidences of DIRs. Recent reports in the literature have revealed an incidence of DIRs to Juvederm Volbella of $1.0 \%$ per patient and $0.8 \%$ per syringe, which is higher than the previously reported incidence of $0.02 \%,{ }^{2}$ and more compatible with the $4.25 \%$ incidence of DIRs to Juvederm Volbella reported by Artzi et al. ${ }^{3}$ It is has been speculated that HA-based fillers with a low-molecular weight-degraded products have higher proinflammatory activity. ${ }^{4}$

DIRs following HA-based filler injections manifest as discoloration (most commonly as erythema), painful nodules, induration or tissue hardening, and solid edema. ${ }^{5,6}$ In past publications, "late" reactions were mostly defined as the ones occurring 14 days to 1 -year post-injection, whereas "delayed" complications were those occurring 1 year or longer after treatment. ${ }^{7-14}$ To clearly differentiate between early and delayed reactions, the panel recommended that 
delayed and late-onset reactions should be considered as one entity under the term delayed inflammatory reactions (DIRs), because their cause is usually not well defined and, more importantly, the initial treatment is similar regardless of etiology. Notably, a DIR arises from a quiescent state 2-4 weeks or longer post-injection.

It is unclear whether DIRs should be considered as true "hypersensitivity" reactions. As such, the panel strongly supported an infectious etiology or trigger, and rejected the word "hypersensitivity" in the context of DIRs.

Triggers that might be associated with the onset of DIRs include viral infection, active sinusitis, low-quality products, combinations of different products, improper technique, past and current dental procedures. ${ }^{8,15}$

Diagnostic tests to guide the management of delayedonset nodules have been proposed in the literature by numerous authors. Most of them support the initial need to rule out a fluctuant nodule that requires incision and drainage of content to be sent for aerobic and anaerobic bacterial, mycobacterial, and fungal cultures before initiating any treatment. ${ }^{7,16-18}$ Of note, the lab must be informed of any suspicion of a mycobacterial process since it can be more challenging to grow and often requires longer culture periods. Polymerase chain reaction (PCR), fluorescent insitu hybridization, and electron microscopy of tissue specimens may be contributory in establishing the infectious agent. ${ }^{19}$ A biopsy and tissue culture should be obtained if there is no resolution following treatment. ${ }^{8,16,17,19}$ Ultrasound imaging is considered as diagnostic "gold standard" in several publications, ${ }^{10}$ based on its abilities to specify the exact location of delayed-onset nodules in relation to other structures, as well as to demonstrate the density of the filler composition. Blood tests for evaluating inflammatory markers, such as C-reactive protein, have also been suggested. ${ }^{8}$

There are numerous treatment options for DIRs, ${ }^{8,20}$ however, stepwise algorithmic approaches for the management of DIRs are lacking. The treatment modalities most frequently described are antibiotic therapy, IL or oral corticosteroids, and hyaluronidase injections. According to the literature, the first-line treatment of choice is antibiotics, ${ }^{7,13,16-22}$ and the use of dual or triple antibiotic therapy is favored. ${ }^{7,13,18-21}$ Several authors recommend combining therapy with broadspectrum antibiotics (ie, fluoroquinolones) and a macrolide, such as clarithromycin, ${ }^{7,17-21}$ to be taken over a period of several weeks. Macrolides are considered especially effective due to their ability to restrain quorum sensing along with their ability to accumulate in adipose tissue, bearing in mind that fillers are mostly located in fat. ${ }^{18}$ Other reported regimens included clarithromycin $500 \mathrm{mg}$ and moxifloxacin $400 \mathrm{mg}$ BID for 10 days, ciprofloxacin 500-750 mg BID for 2-4 weeks, or minocycline $100 \mathrm{mg}$ daily for 6 months. ${ }^{16}$ Antibiotic treatment should be based on results of cultures, and a macrolide may be considered for $2-4$ weeks in the event that no laboratory diagnosis has been reached. Other first-line options include IL hyaluronidase, ${ }^{12,18,19}$ IL steroids, ${ }^{5,22}$ or the combination of both. ${ }^{5,18,22,23}$ Hyaluronidase enables the bond between $\mathrm{C} 4$ of the glucuronic acid and $\mathrm{C} 1$ of the glucosamine to disintegrate, whereupon the HA undergoes hydrolysis. ${ }^{24}$ It is usually recommended to initiate treatment with five units of hyaluronidase. The dose should be doubled in more resistant fillers (eg, the Vycross family by Juvederm). ${ }^{25,26}$ The IL hyaluronidase dosage recommended by a consensus group, ${ }^{17}$ is 10-20 U single injection for an area $<2.5 \mathrm{~mm}$, and $2-4$ injection points with a $10-20 \mathrm{U}$ per injection point for areas from $2.5 \mathrm{~mm}$ to $1 \mathrm{~cm}$ in size. Repeated injections may be necessary in both cases, and higher doses will be required in resistant cases. ${ }^{12}$

The most popular choice for second-line therapy is IL steroids. ${ }^{12,13,16-21,23}$ To further reduce the number of complications secondary to IL steroids as well as temporary skin atrophy, many authors have suggested reconstitution of steroids with other agents, such as 5-FU, lidocaine, or saline. Alternative options for second-line treatments are antibiotic therapy, ${ }^{12,23}$ IL hyaluronidase, ${ }^{7,16,17}$ IL 5-FU, ${ }^{22,23}$ radiofrequency therapy, ${ }^{18,21}$ laser therapy, ${ }^{21,23}$ human platelet-rich plasma, ${ }^{12}$ or extraction of the material with a 16-gauge needle and a syringe while applying negative pressure. ${ }^{22}$ Surgical excision of nodules was considered only as a last resort by many, 7,13,16,17,21,22,24 especially when a granuloma is suspected. ${ }^{12,18,21}$

\section{Panel Recommendations}

This panel has proposed the following therapeutic approach for a DIR. The treating physician must first consider whether the nodules that appear after HA-based filler injections are likely to improve spontaneously, are small in size with no or minimal pain, or, alternatively, if they are relatively large (usually $>0.5 \mathrm{~cm}$ ), not improving, painful, edematous, and erythematous. The former call for a watchful waiting approach, whereas the latter are considered true DIRs and require intervention. It should be considered that small and short-lived edematous nodules are frequently observed in conjunction with or following 


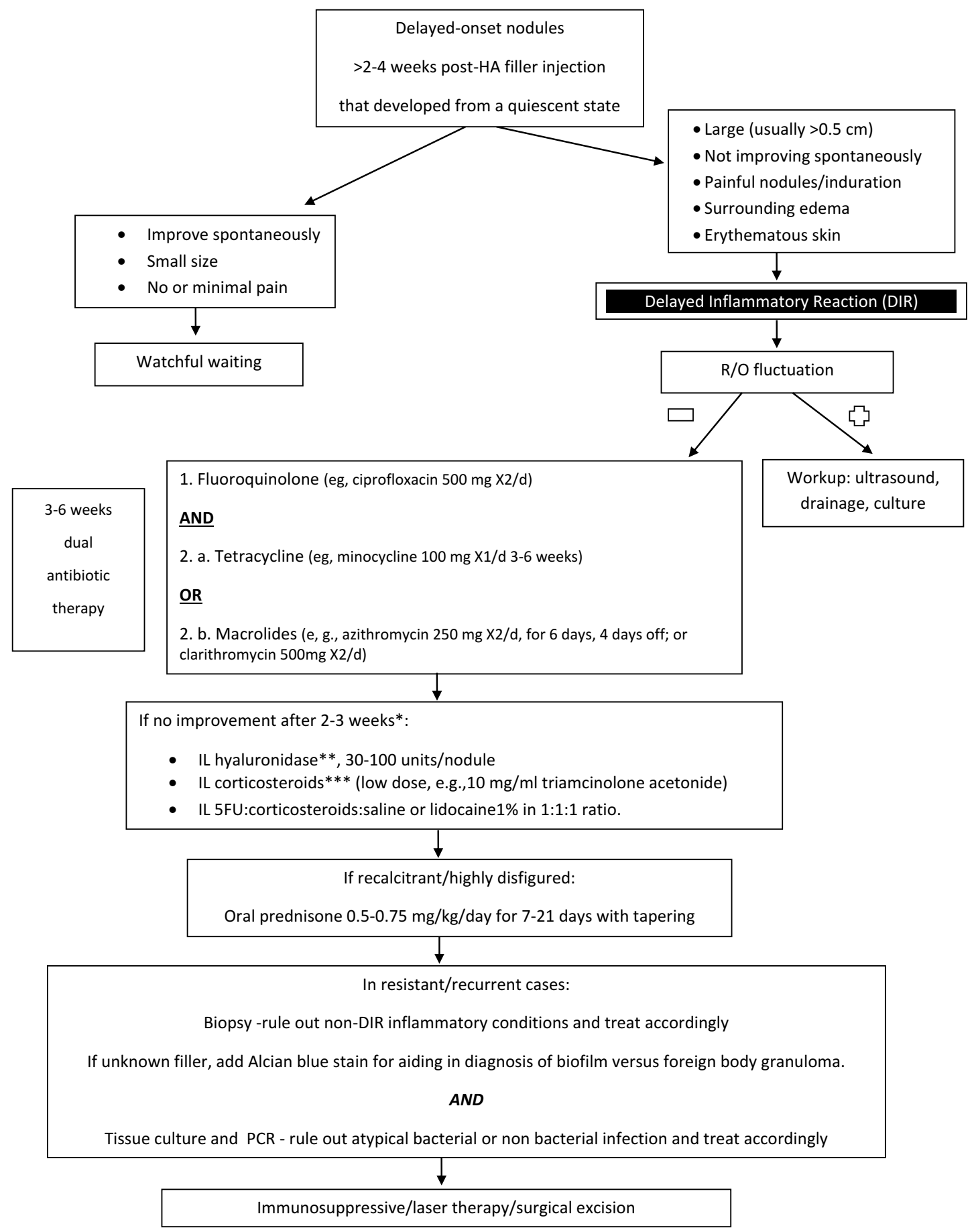

* May be given 24 hours-2 weeks post-antibiotic therapy, and subsequent IL hyaluronidase use may be repeated after 2-3 weeks

${ }^{* *}$ For treating Vycross-associated DIRs, IL hyaluronidase is considered first-line together with dual antiobiotic therapy

${ }^{* * *}$ Can be repeated every 3-4 weeks. Exert great care when treating the periorbital area.

Figure I Algorithm for treating DIRs secondary to HA filler injections. 
a brief illness (eg, viral infections or post-dental procedures) and tend to heal spontaneously.

The second step in the evaluation of a DIR is to consider the presence or absence of fluctuation on palpation. A soft-tissue ultrasound study should be performed for fluctuant nodules, along with incision and drainage. The aspirants should be cultured. Once fluctuation has been ruled out, the first-line treatment of DIRs should consist of antibiotics. The expert panel has divided opinions regarding the appropriate antibiotic regimen, but the majority favor dual antibiotic treatment consisting of a fluoroquinolone (eg, ciprofloxacin $500 \mathrm{mg}$ BID) with either a tetracycline (eg, doxycycline or minocycline $100 \mathrm{mg} /$ day) or a macrolide (eg, clarithromycin $500 \mathrm{mg}$ BID) for a period of 3-6 weeks. Importantly, patients must be informed about a recent correlation that had been found between ciprofloxacin and aortic aneurysms. ${ }^{27}$ Additionally, ciprofloxacin should not be taken with dairy products, and probiotic treatment is highly recommended in order to prevent Clostridium difficile infections resulting from the long duration of antibiotic use. ${ }^{28}$ Furthermore, five of the participating experts recommend avoiding the use of amoxicillin/clavulanic acid or clindamycin for the treatment of DIRs, except in the case of oral cavity or dental-associated infections.

Dissolution of a filler by means of IL hyaluronidase may be postponed by 24 hours to 2 weeks after starting the antibiotic treatment, unless a more resistant HA (ie, Vycross) has been injected, in which case IL hyaluronidase must be given as early as possible. A dose of 30-300 units of IL hyaluronidase should be given per nodule. A fine needle with a low gauge (ie, 18 or $21 \mathrm{G}$ ) is preferred in order to disrupt an encapsulated (filler) organization by allowing for more penetrations. Subsequent dissolution via IL hyaluronidase with increasing dosages should be repeated after 2-3 weeks; however hyaluronidase injections should be limited to 2-3 cycles.

Intralesional steroids alone or combined with 5-FU and saline/lidocaine may be considered for second-line therapy. A low dose of IL corticosteroids should be used to prevent atrophy. The panel recommends the use of a combination of IL triamcinolone $(10-20 \mathrm{mg} / \mathrm{mL}), 5-\mathrm{FU}$, and saline or lidocaine $1 \%$ in a 1:1:1 ratio. It also emphasizes the need for caution when injecting IL corticosteroids into periorbital lesions.

Most participating experts recommended refraining from the use of systemic corticosteroids, with the exception of cases of extremely inflammatory or disfiguring edema and recalcitrant nodules. When oral steroids are prescribed, a low-to-moderate dose and a short-to-medium-term regimen is recommended (ie, $0.5-0.75 \mathrm{mg} / \mathrm{kg} /$ day for $7-21$ days with tapering).
To the best of our knowledge, the current literature does not address the issues of how a physician should approach recurrent DIRs or the risk of developing recurrent DIRs. This led the panel to address two additional questions that physicians may encounter in their daily practice. The first is whether physicians should choose a different therapeutic scheme if a patient returns with a recurrent episode several months after a previous episode has subsided. The second concern how one should pursue future injections: for example, should the same filler technology or brand be used at the same site of injection, and how long should the interval be in relation to the initial reaction? In response to the first question, 16 of the 18-panel members agreed that they would treat recurrent episodes in the same manner as they would an initial episode. Three of them, however, stated that they would make minor adjustments, such as initiating treatment with steroids, increasing the IL hyaluronidase dosage, or using a mixture of IL steroids/5FU/lidocaine. In response to the second question, the panel unanimously agreed they would perform future injections, but with the caveat of opting for a different HA filler technology or a non-HA filler, such as calcium hydroxyapatite, while three panelists opted for the use of fat transfer instead. The recommended waiting time before performing another injection was 3 months to 1-year post-remission in areas other than those where a DIR had occurred, along with performing the procedure with concomitant steroid therapy. Eight panelists advised using smaller quantities of HA and not exceeding a total of $1 \mathrm{cc}$ or more than $0.1 \mathrm{cc}$ per test site for first re-injection post DIR.

\section{Conclusion}

There is a multitude as well as a wide diversity of opinions regarding the management of DIRs in the literature. Our panel emphasizes the need to establish an easy-to-follow and uniform algorithm (Figure 1) for the injecting physician who encounters a DIR.

\section{Disclosure}

Joel L. Cohen, MD has served as a clinical trial participant and/or consultant for Allergan, Galderma, Merz. He reports personal fees from Allergan, grants, personal fees from Galderma, Merz, Revance, and CROMA, during the conduct of the study. Jeffrey S. Dover, MD has served as a consultant and conducted research funded by Allergan, Galderma, Sunera, Merz. Daniel Cassuto, MD is a speaker and trainer for IBSA and MATEX LAB. Vivian Bucay, $\mathrm{MD}$ is a speaker and consultant for Allergan, Galderma, 
and Merz. Tingsong Lim MD is a speaker and trainer for Allergan CoolSculpting, Merz Aesthetics, Sinclair Pharma and Teoxane. Sonja Sattler, MD reports personal fees from Pharm Allergan, MERZ Aesthetics, and CROMA, as a speaker or in research projects, outside the submitted work. Tanja Fischer reports grants from Merz, Croma, Riemser, and PromoItalia, outside the submitted work. Tatjana Pavicic reports personal fees from and is a speaker and consultant for Merz Aesthetics and AAT, outside the submitted work. The authors report no other conflicts of interest in this work.

\section{References}

1. Shalmon D, Cohen J, Landua M, Verner I, Sprecher E, Artzi O. Management of delayed inflammatory reactions to hyaluronic acid fillers: an online survey in Israel. In press 2020.

2. Sadeghpour M, Quatrano NA, Bonati LM, Arndt KA, Dover JS, Kaminer MS. Delayed-onset nodules to differentially crosslinked hyaluronic acids: comparative incidence and risk assessment. Dermatol Surg. 2019;45(8):1085-1094. doi:10.1097/DSS.0000000 000001814

3. Artzi O, Loizides C, Verner I, Landau M. Resistant and recurrent late reaction to hyaluronic acid-based gel. Dermatol Surg. 2016;42 (1):31-37. doi:10.1097/DSS.0000000000000562

4. Farwick M, Gauglitz G, Pavicic T, et al. Fifty-kDa hyaluronic acid upregulates some epidermal genes without changing TNF- $\alpha$ expression in reconstituted epidermis. Skin Pharmacol Physiol. 2011;24 (4):210-217. doi: $10.1159 / 000324296$

5. Lemperle G, Rullan PP, Gauthier-Hazan N. Avoiding and treating dermal filler complications. Plast Reconstr Surg. 2006;118(3S):92S107S. doi:10.1097/01.prs.0000234672.69287.77

6. Kim H, Cho SH, Lee JD, Kim HS. Delayed onset filler complication: two case reports and literature review. Dermatol Ther. 2017;30(5): e12513. doi:10.1111/dth.12513

7. Rohrich RJ, Monheit G, Nguyen AT, Brown SA, Fagien S. Softtissue filler complications: the important role of biofilms. Plast Reconst Surg. 2010;125(4):1250-1256.

8. Beleznay K, Carruthers JD, Carruthers A, Mummert ME, Humphrey S. Delayed-onset nodules secondary to a smooth cohesive $20 \mathrm{mg} / \mathrm{mL}$ hyaluronic acid filler: cause and management. Dermatol Surg. 2015;41(8):929-939. doi:10.1097/DSS.0000000000000418

9. Monheit GD, Rohrich RJ. The nature of long-term fillers and the risk of complications. Dermatol Surg. 2009;35:1598-1604. doi:10.1111/ j.1524-4725.2009.01336.x

10. Cassuto D, Sundaram H. A problem-oriented approach to nodular complications from hyaluronic acid and calcium hydroxylapatite fillers: classification and recommendations for treatment. Plast Reconst Surg. 2013;132(4S-2):48S-58S.

11. Alsaad SM, Fabi SG, Goldman MP. Granulomatous reaction to hyaluronic acid: a case series and review of the literature. Dermatol Surg 2012;38(2Part 1):271-276. doi:10.1111/j.1524-4725.2011.02214.x
12. Urdiales-Gálvez F, Delgado NE, Figueiredo V, et al. Treatment of soft tissue filler complications: expert consensus recommendations. Aesth Plast Surg. 2018;42(2):498-510. doi:10.1007/s00266-017-1063-0

13. Narins RS, COLEMAN III WP, Glogau RG. Recommendations and treatment options for nodules and other filler complications. Dermatol Surg. 2009;1(35):1667-1671. doi:10.1111/j.1524-4725.2009.01335.x

14. Lemperle G, Gauthier-Hazan N. Foreign body granulomas after all injectable dermal fillers: part 2. Treatment options. Plast Reconst Surg. 2009;123(6):1864-1873. doi:10.1097/PRS.0b013e3181858f4f

15. De Boulle K, Heydenrych I. Patient factors influencing dermal filler complications: prevention, assessment, and treatment. Clin Cosmet Invest Dermatol. 2015;8:205. doi:10.2147/CCID.S80446

16. Chiang YZ, Pierone G, Al-Niaimi F. Dermal fillers: pathophysiology, prevention and treatment of complications. J Eur Acad Dermatol Venereol. 2017;31(3):405-413. doi:10.1111/jdv.13977

17. Signorini M, Liew S, Sundaram H, et al. Global aesthetics consensus: avoidance and management of complications from hyaluronic acid fillers - evidence-and opinion-based review and consensus recommendations. Plast Reconst Surg. 2016;137(6):961. doi:10.1097/ PRS.0000000000002184

18. Hartmann D, Ruzicka T, Gauglitz GG. Complications associated with cutaneous aesthetic procedures. J Dtsch Dermatol Ges. 2015;13 (8):778-786.

19. Ibrahim O, Overman J, Arndt KA, Dover JS. Filler nodules: inflammatory or infectious? A Review of biofilms and their implications on clinical practice. Dermatol Surg. 2018;44(1):53-60. doi:10.1097/ DSS.0000000000001202

20. Snozzi P, van Loghem JA. Complication management following rejuvenation procedures with hyaluronic acid fillers - an algorithmbased approach. Plas Reconst Surg Glob Open. 2018;6(12).

21. Kulichova D, Borovaya A, Ruzicka T, Thomas P, Gauglitz GG. Understanding the safety and tolerability of facial filling therapeutics. Expert Opin Drug Saf. 2014;13(9):1215-1226. doi:10.1517/14740338.2014.939168

22. Funt D, Pavicic T. Dermal fillers in aesthetics: an overview of adverse events and treatment approaches. Clin Cosmet Investig Dermatol. 2013;6:295.

23. Ozturk CN, Li Y, Tung R, Parker L, Piliang MP, Zins JE. Complications following injection of soft-tissue fillers. Aesthet Surg J. 2013;33(6):862-877. doi:10.1177/1090820X13493638

24. Rohrich RJ, Ghavami A, Crosby MA. The role of hyaluronic acid fillers (Restylane) in facial cosmetic surgery: review and technical considerations. Plast Reconst Surg. 2007;120(6S):41S-54S. doi:10.10 97/01.prs.0000248794.63898.0f

25. Jones D, Tezel A, Borrell M. In vitro resistance to degradation of hyaluronic acid dermal fillers by ovine testicular hyaluronidase. Dermatol Surg. 2010;36:804-809. doi:10.1111/j.1524-4725.2010.01550.x

26. Vartanian AJ, Frankel AS, Rubin MG. Injected hyaluronidase reduces restylane-mediated cutaneous augmentation. Arch Facial Plast Surg. 2005;7(4):231-237. doi:10.1001/archfaci.7.4.231

27. Pasternak B, Inghammar M, Svanström H. Fluoroquinolone use and risk of aortic aneurysm and dissection: nationwide cohort study. $B M J$. 2018;360:k678. doi:10.1136/bmj.k678

28. Madoff SE, Urquiaga M, Alonso CD, Kelly CP. Prevention of recurrent Clostridioides difficile infection: a systematic review of randomized controlled trials. Anaerobe. 2020;61:102098. 


\section{Publish your work in this journal}

Clinical, Cosmetic and Investigational Dermatology is an international, peer-reviewed, open access, online journal that focuses on the latest clinical and experimental research in all aspects of skin disease and cosmetic interventions. This journal is indexed on CAS.
The manuscript management system is completely online and includes a very quick and fair peer-review system, which is all easy to use. Visit http://www.dovepress.com/testimonials.php to read real quotes from published authors.

Submit your manuscript here: https://www.dovepress.com/clinical-cosmetic-and-investigational-dermatology-journal 